\title{
The Research on a Confined Modeling Method of the Effect of Deformable Ground Soil and Crawler Board
}

\author{
LV Wei-wei ${ }^{1, a}$, LI Zhong-xin and LOU Peng \\ School of Mechanical Engineering, Beijing Institute of Technology, Beijing 100081, China
}

\begin{abstract}
There is a great relationship between the passing capacity of vehicles on the ground deformation road and the properties of confined and shear of ground soil, so it is necessary to establish a model that can reflect the soil pressure of the deformation. Physical significance of classic soil pressure experience parameters model is indeterminate, it was generally obtained by a lot of specific soil test. In this paper a new analysis model was built though introducing the maximum stress value of the crawler board and soil surface on basis of a kind of existing analysis model. This model reveals the relations among the confined characteristics of soil, soil parameters and the geometry of confined crawler board. These soil parameters can be obtained through the conventional soil mechanics test without a large number of specific soil test. Through the contrast experiment and predictions results, this model can effectively predict the sinkage of ground soil under the load, and provide a theory basis for the prediction of vehicle mobility.
\end{abstract}

\section{Introduction}

The passing property of vehicles on the soft ground is closely related with soil pressure and shear resistance characteristics. In the study of vehicle ground mechanics, the empirical model is usually used to describe the confined characteristics of the soil. The most representative model is the model [1-2] made by M.G.Bekker. The model is based on the test of vehicle and soil parameters. The simplified equation is used to establish the relationship between the external load and the subsidence deformation of the soil, which provides a theoretical basis for vehicle performance testing. However, the physical meaning of the empirical parameters of the model is not clear. It is necessary to determine the parameters through a large number of expensive and time-consuming soil test tests, and the model is limited to a particular type of model. In recent years, many scholars have established a number of new empirical models [3-4], hoping that the model parameters are not dependent on specific soil tests, but the results show that these model parameters are still directly or indirectly related to the parameters of the Bekker model, and in essence the empirical parameters of the model need to be obtained through a large amount of specific pressure plate subsidence soil tests. Kacigin, Ageikind [5] establish an analytical model, including the external load of soil, the geometric size of the plate, the subsidence of soil and the mechanical properties of soil, which reflects the relationship between the amount of soil subsidence, the load of the soil and the stress in the soil, in which the model parameters can be obtained through the conventional soil mechanics test without a large number of specific soil test. It is only the model that considers the external load applied by the plate / wheel as the uniformly distributed load, which results in inaccurate prediction of the model.

\footnotetext{
${ }^{\text {a }}$ Corresponding author : lvweiwei@bit.edu.cn
} 
On the basis of the analysis model, considering the influence of the soil stress on the maximum stress on the contact surface of the Caterpillar board and the soil, a new analysis model is established to establish the relation between the soil subsidence and the size of the load, the parameters of soil characteristics and the size of the plate, and the test method of the model parameters is given, and the test is tested. It is reasonable to predict the subsidence of soil by the model, which provides a theoretical basis for studying the subsidence deformation of deformable ground soil under the action of vehicle.

\section{The influence of contact stress between track plate and soil on normal stress}

Ageikind et al [6] believes that the normal stress at the $\mathrm{z}$ depth of soil is related to the geometric characteristics of the plate and the uniform load of the contact surface, as shown in formula (1) and (2).

$$
\begin{aligned}
\sigma_{z} & =\frac{J \cdot p}{1+\left(\frac{z}{A_{0}+B}\right)^{2}} \\
\sigma_{z} & =\frac{J \cdot p}{1+\left(\frac{z}{A_{0}+B}\right)^{2}}
\end{aligned}
$$

$\sigma_{z}$ - The internal positive stress of the soil in depth is $\mathrm{Z} ; J$ - The dimensionless coefficient , is related to the size of the plate, $J=\frac{0.03+\frac{L}{B}}{0.6+0.43 \cdot \frac{L}{B}} ; p$-the uniformly distributed load of the soil on the plate, $k p a ; z$-The depth of the soil, $m ; A_{0}$ - The coefficient of quantity, is related to the width of the plate and the depth of the soil; $B$-The smallest straight diameter or width of the plate, $m$.

The recent research shows that the stress in the soil is related to the maximum stress of the contact surface, not the average stress of the contact surface. Therefore, the maximum stress of the contact surface is introduced in this paper to amend the analysis model proposed by Ageikind and to establish a new analytical model, such as formula (3).

$$
\sigma_{z}=\frac{J \cdot p_{\max }}{1+\left(\frac{z}{A_{0}+B}\right)^{2}}
$$

$p_{\max }$ - The maximum stress on the contact surface of the plate to the soil, $k p a$.

According to the classical soil mechanics[7], the maximum stress on the contact surface between the plate and the soil is 3 times that of the average stress on the contact surface, and the relationship between them is shown by formula (4).

$$
p_{\max }=\xi \cdot p
$$

$\xi$ is a dimensionless constant, independent of the state of the soil, and only related to the width 
of the plate, $\xi=1+1.86 \cdot\left(\frac{x}{2}+\frac{x^{2}}{3}-\frac{x^{3}}{5}\right), x=2.5 \cdot(1-\exp (-3.74 \cdot B))$. By calculating the above formula, we can get the normal normal stress $\sigma_{z}$ of the soil in the soil depth of $Z$ under the load.

A circular plate with a diameter of $0.3 m$ is placed horizontally in a soil trough with a soil depth of $1 m$.The soil trough is filled with dry sand, the uniform load $p=21.2,42.5,63.7,85.0$ and 106.2kpa is applied on the circular plate, and the soil normal stress at the soil's depth of $0.2,0.35$ and $0.5 m$ is tested. The results of the test and prediction results of the analysis model (1-2) are shown in Figure 1.

In Figure 1, the soil normal stress at depth of 0.2,0.35and $0.5 \mathrm{~m}$ predicted by Beck's empirical model and analytical model are shown under $p=21.2,42.5,63.7,85.0$ and 106.2kpa in the dotted line and dashed line from left to right. The dotted line represents the results of the soil internal normal stress predicted by Beck's empirical model, and the solid line indicates the normal stress in the soil obtained by the new analytical model. The point in the map indicates the soil normal stress measured by the depth of $0.2,0.35$ and $0.5 \mathrm{~m}$ the pressure sensor under the different uniform load test. It's known that the results of the analytical model are in good agreement with the test results from Figure 1, which indicates that the normal stress in the soil is related to the maximum stress value of the caterpillar plate and the soil contact surface, and can be used to modify the soil pressure characteristic model.

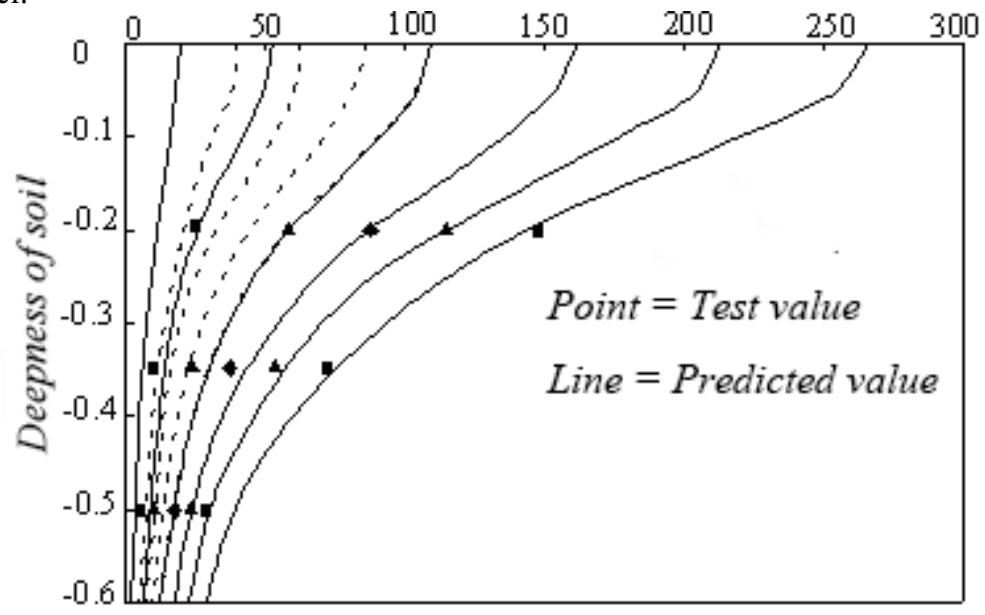

Normal stress of $0.3 m$ diameter for plate, $k p a$

Figure 1. Press plate test

\section{Soil pressure characteristic model}

According to the dynamic soil theory, the force of the crawler and the soil is shown in Figure 2, and the normal pressure on the vertical crawler and the passive earth pressure $\sigma_{p}$ the depth of $Z$ below the a point can be expressed by the formula (5) [8]. 


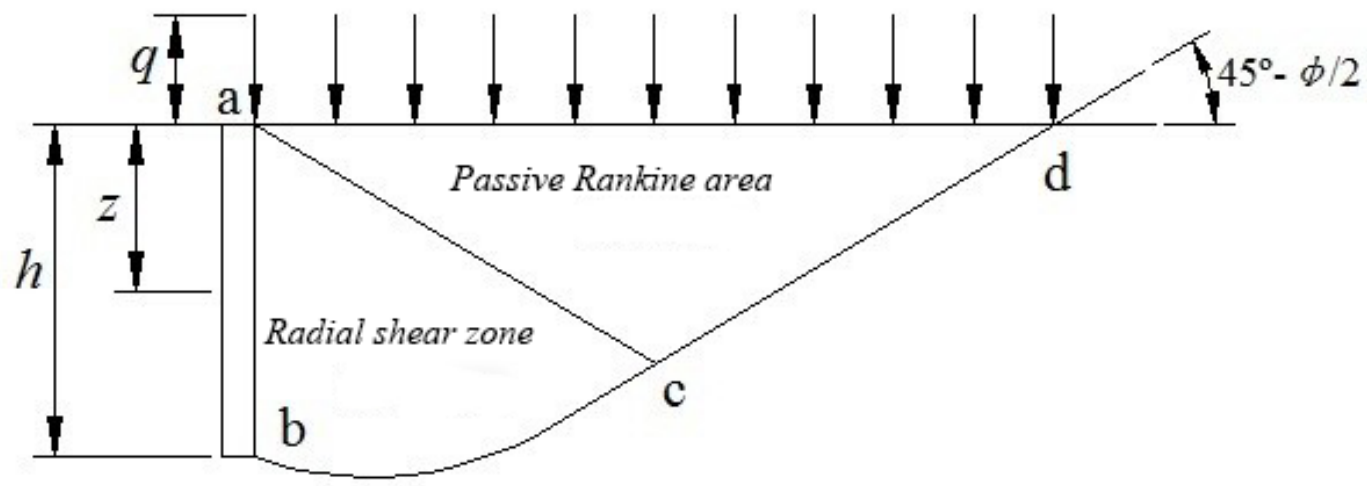

Figure 2. Caterpillar and soil action

$$
\sigma_{p}=\left(N_{\gamma} \cdot n_{\gamma} \cdot B+N_{p} \cdot n_{p} \cdot z\right) \cdot \frac{\gamma \cdot g}{1000}+N_{c} \cdot n_{c} \cdot c
$$

Where: $N_{\gamma}=\exp (6.65 \cdot \tan \phi-1.75) ; N_{p}=\exp (4.76 \cdot \tan \phi+0.15)$;

$$
N_{c}=\exp (3.32 \cdot \tan \phi+1.5) ; n_{\gamma}=1+0.25 \cdot \frac{B}{L} ; n_{P}=1+1.5 \cdot \frac{B}{L} ; n_{c}=1+0.3 \cdot \frac{B}{L} \text {. }
$$

$\sigma_{p}$ — The bearing capacity of soil, kpa; $N_{\gamma}, N_{p}, N_{c}$ — The coefficient of soil carrying capacity, dimensionless; $\gamma$ - The severe of soil, $\mathrm{kg} / \mathrm{m}^{3} ; n_{\gamma}, n_{p}, n_{c}$ - Scale effect coefficient of plate; $g-$ Gravity acceleration $9.8 \mathrm{~m}^{2} / \mathrm{s} ; \mathrm{c} \longrightarrow$ Soil cohesion, $k p a ; \phi \longrightarrow$ Soil friction angle.

According to the theory of soil mechanics limit equilibrium, the stress state of any point in the soil can be expressed by the Mohr stress circle in Figure 3. The shear strength law of the soil is expressed by the Kulun strength line. The conditions of the Mohr circle tangent to the Kulun strength line reflect the limit equilibrium condition of the soil very vividly.

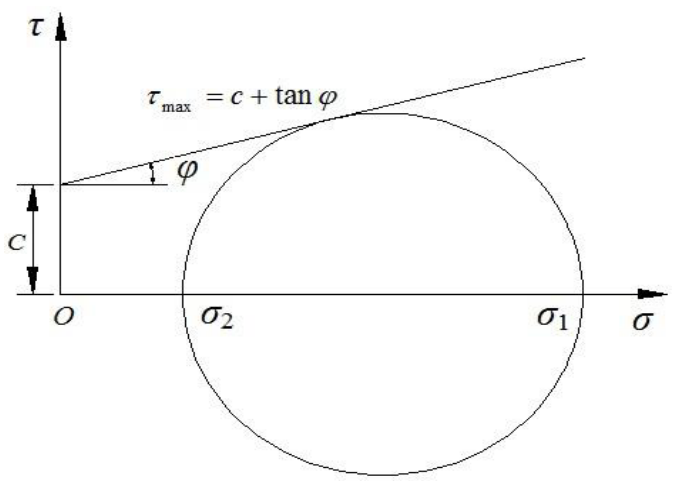

Figure 3. Mole Kulun damage criterion

According to the geometric relationship between the stress circle and the Coulomb strength line in the limit equilibrium state, the relationship between the principal stress of soil material and the maximum shear stress of soil is established.

$$
\sigma_{1}=\sigma_{3} \tan ^{2}\left(45^{\circ}+\frac{\phi}{2}\right)+2 c \tan \left(45^{\circ}+\frac{\phi}{2}\right)
$$


By the limit equilibrium theory, the vertical stress $\sigma_{z}$ on the unit area in the soil depth of $z$ is the same, it is the minimum principal stress $\sigma_{3}$, but the horizontal stress $\sigma_{x}$ is gradually increased and it is the maximum principal stress $\sigma_{1}$ when the limit is reached. It is the dynamic soil pressure strength $\sigma_{p}$.

The analysis model of soil pressure characteristics will be established by introducing $\sigma_{z}$ and $\sigma_{p}$ to the formula (6), as shown in formula (7).

$$
p=\frac{\left(N_{\gamma} \cdot n_{\gamma} \cdot B+N_{p} \cdot n_{p} \cdot z\right) \cdot \frac{\gamma \cdot g}{1000}+N_{c} \cdot n_{c} \cdot c-2 c \tan \beta}{J \cdot \tan ^{2} \beta}\left[1+\left(\frac{z}{A_{0}+B}\right)^{2}\right]
$$

Where $\beta=45^{\circ}+\frac{\phi}{2}$.

The model includes uniform pressure, soil subsidence, plate size and soil properties parameters(cohesive force, internal friction angle and soil gravity). The parameters of the model can be obtained by conventional soil mechanics experiments without a large number of time-consuming and expensive specific soil experiments.

\section{Test verification}

The soil parameters are $w=2.3, c=0, \phi=33^{\circ}$.It's filled with dry sand in the soil trough, and circular plates witha diameter of $0.3 \mathrm{~m} 2.5 \mathrm{~cm}, 5 \mathrm{~cm}, 10 \mathrm{~cm}, 15 \mathrm{~cm}$ and $30 \mathrm{~cm}$ are separatelyput into the soil trough to carry out the pressure plate test. The solid line 1 to 5 in Figure 4 indicates circular platessinkage of the diameter of $0.3 \mathrm{~m} 2.5 \mathrm{~cm}, 5 \mathrm{~cm}, 10 \mathrm{~cm}, 15 \mathrm{~cm}$ and $30 \mathrm{~cm}$ predicted by the analytical model respectively, and the point indicates the soil trough test data. It can be seen the consistency of the prediction results of the analytical model and the experimental results is good from Figure 4. The rationality of predicting the subsidence of soil by the model is verified by experiments, which provides a theoretical basis for the research on vehicle mobility.

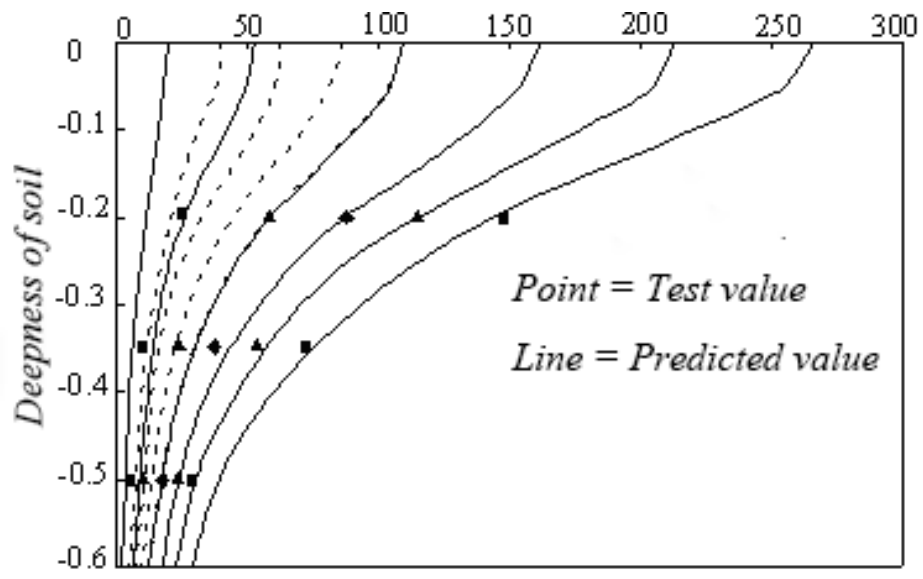

Normal stress of $0.3 \mathrm{~m}$ diameter for plate, $\mathrm{kpa}$

Figure 4. Comparison between experimental results and model prediction results 


\section{Conclusion}

Based on the dynamic soil pressure and limit equilibrium theory, this paper analyzes the interaction relationship between the crawler plate and the ground soil, and establishes an analysis model of the soil pressure characteristics. The model establishes the relationship between the geometric parameters of the crawler plate, the soil subsidence, the external load and the vehicle driving parameters, and These soil parameters can be obtained through the conventional soil mechanics test without a large number of complex and specific soil test. It's discovered that the prediction results of the model are basically the same as those of the test results by comparison with test results, which provides a theoretical basis for predicting the mobility of vehicles.

\section{References}

1. Bekker MG. Theory of land locomotion. Ann Arbor (MI): Universityof Michigan Press; 1956.

2. Bekker MG. Off-the-road locomotion. Ann Arbor (MI): University ofMichigan Press; 1960.

3. Guskov VV. Optimum parameters of agricultural tractors. Moscow:Mashinostroenie; 1966 [in Russian].

4. Kacigin VV, Guskov VV. The basis of tractor performance theory. JTerramech 1968;5(3).

5. Ageikin JS. Off-the-road wheeled and combined traction devices:theory and calculations. New Delhi: Amerind Pub. Co.; 1987 [translation from 1972 Russian book].

6. Ageikin JS. Off-the-road mobility of automobiles. New Delhi:Amerind Pub. Co.; 1987 [translation from 1981 Russian book].

7. Tsytovich NA. Soil mechanics. Moscow: Gosstroizdat; 1963 [inRussian].

8. Modest Lyasko,LSA model for sinkage predictions. Journal of Terramechanics, 47 (2010) 1-19. 